\title{
Bestimmung der Schadstofffrachten an Kontrollebenen mithilfe von Punktkonzentrationsmessungen und Immissionspumpversuchen - ein Vergleich
}

\author{
Maria Herold · Thomas Ptak • Peter Grathwohl
}

Eingang des Beitrages: 22.4.2008/Eingang des überarbeiteten Beitrages: 15.8.2008/Online veröffentlicht: 28. 10.2008

(C) Die Autoren 2008. Dieser Artikel wurde mit Open Access auf Springerlink.com veröffentlicht.

\begin{abstract}
Kurzfassung Die Quantifizierung der Schadstofffrachten an Kontrollebenen ist eine entscheidende Voraussetzung zur Bewertung von Grundwasserschadensfällen und zum Nachweis von Natural Attenuation. Hierzu können zwei verschiedene Erkundungsansätze, die Frachtbestimmung basierend auf der Interpolation von Punktkonzentrationsmessungen sowie Immissionspumpversuche, verwendet werden. Punktkonzentrationsmessungen haben den entscheidenden Nachteil, dass in der Regel nur eine ausreichend große Anzahl sicher stellen kann, dass die gesamte Abstromfahne erfasst wird. Immissionspumpversuche können wiederum relativ hohe Grundwasseraufbereitungs- und Entsorgungskosten verursachen und nur in ausreichend durchlässigen Grundwasserleitern durchgeführt werden. Ein Vergleich der Ergebnisse aus der Anwendung beider Erkundungsansätze kann eine Hilfestellung bei der Konzipierung von Erkundungsmaßnahmen bieten. Daher wurden die Resultate von vier Immissionspumpversuchen mit Messungen in 13, in kurzem Abstand zueinander errichteten, Direct-Push-Messstellen auf dem Gelände eines ehemaligen Gaswerks verglichen. Es konnte festgestellt werden, dass die Vergleichbarkeit der Erkundungsergebnisse je nach Standortsituation stark von der Heterogenität der Verteilung der Schadstoffe im Grundwasserleiter abhängt. Die Studie legt nahe, dass insbesondere bei stark heterogenen Verhältnissen im Grundwasserleiter Immissionspumpversuche bei der Wahl
\end{abstract}

M.Sc. M. Herold ( $\bowtie)$, Prof. Dr.-Ing. T. Ptak

Angewandte Geologie, Geowissenschaftliches Zentrum,

Universität Göttingen, Goldschmidtstr. 3, 37077 Göttingen

E-Mail:mherold@gwdg.de, thomas.ptak@geo.uni-goettingen.de

Prof. Dr. P. Grathwohl

Zentrum für Angewandte Geowissenschaften, Universität Tübingen,

Sigwartstr. 10, 72076 Tübingen

E-Mail: peter.grathwohl@uni-tuebingen.de der Erkundungsmethode bevorzugt werden sollten, da die Interpolation von Punktkonzentrationsmessungen selbst im Falle eines relativ engmaschigen Messstellennetzes zu einer großen Erkundungsunsicherheit führen kann.

\section{A comparison of mass flow rate estimates based on point scale and integral measurements}

\begin{abstract}
The quantification of contaminant mass flow rates at control planes is an essential prerequisite for assessing contaminated sites and for providing evidence of natural attenuation. Two different investigation approaches are usually implemented: mass flow estimation based on interpolation of point scale concentration measurements, and integral pumping tests. Point scale concentration measurements have the crucial disadvantage that in general, only a sufficiently dense monitoring network can ensure that the plume is completely covered. On the other hand, integral pumping tests may require expensive groundwater treatment and disposal and are only applicable in sufficiently conductive aquifers. A comparison of results from the application of both approaches can help with respect to the selection of a subsurface investigation method. A former gasworks site was chosen to compare the results of four integral pumping tests and measurements obtained from 13 direct-pushwells, which were installed at a relatively close spacing. The comparison shows that the correlation of the two methods depends strongly on the heterogeneity of the contaminant distribution within the aquifer. The study suggests that especially in the case of heterogeneous settings, integral pumping tests should be chosen for subsurface investigations, as interpolated point scale concentration measurements, even if densely spaced, can still bear a prohibitively high degree of uncertainty.
\end{abstract}


Keywords contaminant mass flow rate $\cdot$ integral pumping tests ' point scale concentration measurements $\cdot$ remediation optimization

\section{Einleitung}

Die Quantifizierung von Schadstofffrachten und der Abnahme der Schadstofffrachten zwischen zwei oder mehreren Kontrollebenen ist ein essentieller Bestandteil der Beurteilung von Schadensfällen in Grundwasserleitern, insbesondere wenn Natural Attenuation (NA) im Rahmen der Sanierungsplanung in Betracht gezogen wird. Sowohl das LABO-Positionspapier (2005) als auch die US-EPA (2004) betonen für die NA-Untersuchung die Notwendigkeit einer Charakterisierung der Schadenssituation, welche sowohl die räumliche als auch die zeitliche Ausbreitung der Schadstofffahne und den Austrag aus der Schadstoffquelle betrachtet. Die US-EPA betont insbesondere die möglichst dreidimensionale Charakterisierung der Fahne sowie die Beantwortung der Frage, ob es sich um eine stationäre oder instationäre Schadstofffahne handelt. Erst bei Vorliegen dieser Informationsgrundlage, welche sicher stellen muss, dass die gesamte Fahne erfasst wurde, kann es zu einer Einschätzung und Bewertung des Schadensfalls sowie der genauen Konzeption, Positionierung und Dimensionierung von Sanierungsmaßnahmen und der eventuellen Berücksichtigung von NA kommen. Dafür können die berechneten Schadstofffrachten als Eingangsgrößen für ein reaktives Strömungs- und Transportmodell verwendet werden, mit dessen Hilfe das NA-Potenzial des Standorts evaluiert und mögliche Sanierungsstrategien entwickelt werden. Ein Beispiel für die Bestimmung des NA-Potenzials durch Berechnung der Schadstofffrachten von Benzol, Toluol, Ethylbenzol und Xylol (BTEX) und Polyaromatischen Kohlenwasserstoffen (PAKs) an mehreren hintereinander liegenden Kontrollebenen findet sich z. B. bei Bockelmann et al. (2001) und Bayer-Raich et al. (2006).

Weitere Anwendungsgebiete für die Berechnung der Schadstofffrachten ergeben sich (i) bei der Bewertung unterschiedlicher Schadstoffe in Bezug auf ihre Umweltverträglichkeit und damit deren Priorisierung (Einarson \& Mackay 2001), sowie (ii) bei der Beurteilung von Sanierungsmaßnahmen an Schadstoffquellen (Soga et al. 2004).

Sowohl die Zusammensetzung und Heterogenität der Schadensquelle als auch die Heterogenität des betroffenen Grundwasserleiters sind ausschlaggebend für die Freisetzung und Verteilung der Schadstoffe im Untergrund. In der Regel resultiert hieraus eine ungleichmäßige Verteilung der Schadstoffmasse innerhalb der Schadstofffahne. Erfahrungsgemäß ist die Berechnung der Schadstofffrachten an Kontrollebenen, insbesondere aufgrund möglicher Instationarität des hydraulischen Systems, keine triviale Aufgabe.
Prinzipiell lassen sich hierzu zwei verschiedene Erkundungsansätze einsetzen, die Frachtbestimmung basierend auf der Messung von Punktkonzentrationswerten und deren Interpolation sowie der räumlich integrierende Ansatz, der zur Entwicklung von Immissionspumpversuchen (IPV) geführt hat. Ersterer umfasst neben der Messung von Konzentrationen an einzelnen Messstellen bzw. Brunnen auch die Bestimmung der hydraulischen Leitfähigkeit und des hydraulischen Gradienten, optimalerweise an jedem Messpunkt, wobei manche Interpolationsansätze tiefenhorizontierte Parameter benötigen. Abhängig von der Anzahl der Messstellen können dabei hohe Erkundungskosten entstehen, wobei eine relativ geringe Anzahl von Messstellen den entscheidenden Nachteil hat, dass nur ein relativ kleiner Teil des Untergrundes mit seiner Schadstoffverteilung direkt erfasst wird. Immissionspumpversuche stellen eine weitergehende Erkundungsmaßnahme dar, die mit einer umfassenden Vor- und Nachbereitung und je nach Standortsituation mit ggf. relativ hohen Wasseraufbereitungs- und -entsorgungskosten verbunden ist. IPV haben den Vorteil, dass weitere relevante Parameter wie z. B. die Transmissivität in einem Schritt erfasst werden und ermöglichen gleichzeitig die vollständige Erfassung der Schadstofffahne und somit eine verlässliche Berechnung der Schadstofffrachten. Hier kann (i) ein Vergleich der Ergebnisse aus der Anwendung beider Erkundungsansätze Stärken und Limitationen der Methoden aufzeigen und (ii) eine Analyse der methodentypischen Unsicherheiten ermöglichen, Empfehlungen zum Einsatz dieser Methoden geben zu können.

Bisher sind mehrere verschiedene Vorgehensweisen, deren Ziel die Bestimmung des Massenflusses durch Interpolation von Punktkonzentrationsmessungen war, vorgestellt worden, wobei hier insbesondere die Unterschiede im Umfang und Qualität der Datengrundlage von Bedeutung sind. King et al. (1999), Borden et al. (1997) sowie Kao \& Wang (2001) verfolgten aufgrund der guten Datengrundlage einen Ansatz, bei dem alle Parameter sowohl horizontal als auch vertikal aufgelöst in die Berechnung eingehen konnten. Hatfield et al. $(2002,2004)$ verwendeten Fluxmeter, um den spezifischen Massenfluss direkt an der Messstelle $\mathrm{zu}$ bestimmen. Einen ähnlichen Ansatz verfolgte Ballard (1996), der mit einem Durchflusssensor die Richtung und Stärke des Grundwasserflusses in drei Dimensionen bestimmte. Eher klassische Methoden setzten Newell et al. (2003) und Bockelmann et al. (2003) ein, wobei sich ihre Arbeiten durch die horizontale und vertikale Auflösung der verwendeten Datensätze unterscheiden. Bei Kübert \& Finkel (2006) findet man einen Vergleich dieser verschiedenen Ansätze sowie die Empfehlung, bei zunehmend heterogenen Verhältnissen integrierende Methoden zu verwenden. Die Heterogenität des Untergrunds führt in der Regel zu stark unregelmäßig ausgebildeten Schadstofffahnen, wobei die Qualität der Bestimmung der Schadstofffracht wesent- 
lich von der Anzahl und Positionierung der MonitoringMessstellen abhängt. Ebenso bewirkt eine heterogene Verteilung der Schadstoffe im Schadensherd ungleichmäßig verteilte Schadstoffkonzentrationen im Abstrom. Nur ein ausreichend dichtes Messstellennetz könnte sicherstellen, dass die Fahne in ihrem ganzen Ausmaß erfasst wird. Da eine solche Erkundungsmaßnahme schnell an ihre finanziellen Grenzen stoßen kann und daher in der Regel keine ausreichende Erkundungssicherheit bieten kann, wurden räumlich integrierende Immissionspumpversuche (Teutsch et al. 2000, Ptak et al. 2000) entwickelt, die, angeordnet entlang einer oder mehrerer Kontrollebenen, im Abstrom des Schadensherdes durchgeführt werden. Hierbei handelt es sich um eine nicht notwendigerweise günstigere Erkundungsmaßnahme, die aber eine höhere Aussagesicherheit bietet, da sich durch das große Erfassungsvolumen an den einzelnen IPV-Brunnen die aus der räumlichen Konzentrationsvariabilität resultierende Erkundungsunsicherheit reduziert. Immissionspumpversuche basieren auf der Auswertung von Schadstoffkonzentrationsganglinien $(\mathrm{C}(\mathrm{t}))$, die während eines Pumpversuchs durch Beprobung des Grundwassers gewonnen werden können. Durch Anwendung eines Inversionsalgorithmus auf die gemessenen Schadstoffkonzentrationsganglinien erhält man dann eine räumliche Konzentrationsverteilung $(\mathrm{C}(\mathrm{x}))$ entlang einer Kontrollebene und daraus die gesamte Schadstofffracht und die mittlere Schadstoffkonzentration an der Kontrollebene. Hierbei besteht die Schwierigkeit sicherzustellen, dass die gesamte Fahnenbreite erfasst wird. Falls nicht örtliche Gegebenheiten am Standort (z.B. die Position der seitlichen Aquiferbegrenzung, wie beim hier nachfolgend betrachteten Beispiel) oder IPV ohne messbare Schadstoffkonzentration diese Unsicherheit reduzieren, können dies Punktkonzentrationsmessungen bewirken.

Die Inversion kann analytisch, unter Berücksichtigung der natürlichen Grundwasserströmung (Bayer-Raich et al. 2004) und numerisch, unter Einbindung eines Grundwasserströmungs- und Transportmodells (Bayer-Raich et al. 2003) erfolgen.

Bei der analytischen Lösung wird von einem homogenen Grundwasserleiter mit stationärer Grundwasserströmung ausgegangen, wohingegen die numerische Lösung in Verbindung mit einem Strömungs- und Transportmodell heterogene geologische Verhältnisse sowie instationäre Grundwasserströmung berücksichtigt. Bayer-Raich et al. (2007) konnten zeigen, dass Konzentrationsgradienten, so weit sie parallel zur Grundströmung linear verlaufen, innerhalb des Entnahmebereichs des IPV keinen signifikanten Einfluss auf die Auswertung haben, sodass bei beiden Lösungen ein Transport ohne Abbau angenommen werden kann. Beispiele für solche Auswertungen findet man unter anderem bei Bockelmann et al. (2001, 2003), Bauer et al. (2004) und Rügner et al. (2004), wobei Ptak et al. (2004) eine höhere räumliche Auflösung durch vertikale Differenzierung der IPV-Konzentrationsganglinien erreicht haben.

In der vorliegenden Studie werden beide Erkundungsansätze zur Quantifizierung von Schadstofffrachten an einem ehemaligen Gaswerksstandort (Testfeld Süd, Herfort et al. 1998) erstmals unter Feldbedingungen quantitativ miteinander verglichen, um ihre Eignung zur Erkundung heterogener Aquifere beurteilen zu können.

\section{Standortbeschreibung}

Hydrogeologie

Das Testfeld Süd befindet sich auf dem Gelände eines ehemaligen Gaswerksstandortes im Neckartal in Süddeutschland und hat eine Nord-Süd-Ausdehnung von ungefähr $1.000 \mathrm{~m}$ und eine Breite zwischen 160 und $400 \mathrm{~m}$. Es wird im Osten durch den Neckar und im Westen durch einen hydraulisch verhältnismäßig undurchlässigen Talrand begrenzt, der aus mesozoischen Mergeln und intermediären Hanglehmschichten besteht.

Der hier untersuchte quartäre Grundwasserleiter mit einer Mächtigkeit von durchschnittlich 3,3 m besteht aus Mittelkies mit örtlich begrenzten feinkörnigen Lagen und wird entweder von Auesedimenten oder anthropogenen Auffüllungen überdeckt. Im Liegenden folgt eine $30 \mathrm{~m}$ mächtige Gipskeuperschicht und daraufhin der Obere Muschelkalk. Erstere stellen die Quelle von sulfatreichem Wasser dar, welches im untersuchten Grundwasserleiter nachgewiesen wurde. Die ausgeprägte Heterogenität des Grundwasserleiters ist durch die Sedimentationsbedingungen vor Ort, die Lage am Talrand (Ausstreichen und Auskeilen der Schichten), lokale Einsenkungen im Festgesteinsuntergrund (Störungen und Auslaugung) sowie anthropogene Einflüsse wie Baumaßnahmen (Aushub, Fundamente) bedingt.

Der Grundwasserflurabstand beträgt im Mittel 4,1 m, wobei der Grundwasserleiter, abhängig von der Art der Überdeckung (Auesediment oder anthropogene Auffüllung), gespannt bzw. ungespannt ist.

Die gemessenen Grundwasserpotenziale der letzten 10 Jahre schwanken nur geringfügig, und ein saisonaler Trend ist nicht erkennbar. Beides ist auf die regulierende Funktion des künstlich aufgestauten Vorfluters zurückzuführen. Erkundungsmaßnahmen ergaben einen Gradienten von 0,002 bei einer nach N-NW ausgerichteten Hauptströmungsrichtung (Herfort 2000) sowie eine mittlere Abstandsgeschwindigkeit von $2 \mathrm{md}^{-1}$ (Bösel et al. 2000). Mit Tracerversuchen im Jahr 2007 wurde eine speichernutzbare Porosität von $16 \%$ bestimmt (Trötschler et al. 2007).

Eine große Anzahl von Pumpversuchen im zentralen und südlichen Teil des Geländes ergab einen mittleren $\mathrm{K}_{\mathrm{f}}$-Wert von $3,3 \cdot 10^{-3} \mathrm{~ms}^{-1}$ (Herfort 2000), und aus der hydrauli- 
schen Auswertung der Immissionspumpversuche in den Jahren 2004 und 2006 resultierte ein mittlerer $\mathrm{K}_{\mathrm{f}}$-Wert von $3,7 \cdot 10^{-3} \mathrm{~ms}^{-1}$ für den nördlichen Teil.

Das Gaswerk war zwischen 1875 und 1970 in Betrieb, und Leckagen in Bereichen der Kohleverarbeitung, insbesondere aber die Zerstörungen im 2. Weltkrieg, haben zu einer großen Anzahl von Schadensherden geführt (Zamfirescu \& Grathwohl 2001). Aus diesem Grund wird das Alter des Hauptschadensherdes auf ungefähr 60 Jahre geschätzt. Das Schadstoffspektrum umfasst gaswerkstypische Substanzen wie monoaromatische Kohlenwasserstoffe, polyzyklische aromatische Kohlenwasserstoffe (PAK), aliphatische Kohlenwasserstoffe, Phenole und heterozyklische aromatische Kohlenwasserstoffe (HET) (Zamfirescu 2000). In zahlreichen Bohrungen im südlichen Teil des Testfeldes wurde im Kapillarsaumbereich und an der Basis des Grundwasserleiters Öl in Phase gefunden. Weitere Bohrungen im zentralen und nördlichen Teil weisen auf die Ausbildung einer komplexen Schadstofffahne hin. Die vorherrschenden Bedingungen im Grundwasser sind sulfatreduzierend.

\section{Methodik}

Grundlagen zu Immissionspumpversuchen

Räumlich integrierende Immissionspumpversuche (IPV, Teutsch et al. 2000, Ptak et al. 2000) werden seit einigen Jahren eingesetzt, um Schadstofffrachten unterstromig von Schadstoffquellen zu bestimmen. Hierzu werden Pumpbrunnen entlang einer Kontrollebene angeordnet und entweder gleichzeitig oder sequenziell bepumpt. Die Positionierung der Brunnen sowie deren Pumpraten und Pumpdauer müssen so gewählt werden, dass optimalerweise der gesamte $\mathrm{Ab}$ - strombereich der Verdachtsfläche erfasst wird. Hierfür sind a priori Kenntnisse über die Aquifergeometrie, $\mathrm{K}_{\mathrm{f}}$-Werte, Porositäten und Gradienten, bzw. ein Strömungs- und advektives Transportmodell notwendig. Während der Pumpmaßnahmen werden Konzentrationsganglinien der Schadstoffe und anderer relevanter Grundwasserqualitätsparameter gemessen, die Information über die Position und die Ausmaße der Fahne sowie über die Konzentrationen der Schadstoffe und die Verteilung der Grundwasserparameter geben. Die Konzentrationsganglinien werden dann unter Verwendung eines instationären Inversionsalgorithmus analytisch oder numerisch mithilfe eines Strömungs- und Transportmodells ausgewertet, um Konzentrationsverteilungen entlang der Kontrollebene und daraus Schadstofffrachten zu erhalten. Dieses Prinzip wird in Abbildung 1 verdeutlicht.

\section{Numerische Auswertung}

Der Inversionsalgorithmus, der zur numerischen Inversion der Konzentrationsganglinien verwendet wird, ist in dem Programm CSTREAM (Bayer-Raich et al. 2003, BayerRaich 2004) implementiert, welches eine Erweiterung der Inversionslösung von Schwarz (2002) darstellt. CSTREAM benötigt mindestens ein instationäres Strömungs- und advektives, nicht-reaktives Transportmodell des Untersuchungsgebietes, welches dazu dient, die Stromlinien und Isochronen zu bestimmen. Dieses Modell simuliert alle IPV unter Berücksichtigung der räumlichen Variabilität der hydraulischen Leitfähigkeit, der Porosität und der Mächtigkeit des Grundwasserleiters, wobei diese Parameter durch mehrere Pumpversuche, Markierungsversuche bzw. Bohrungen bestimmt wurden. Dadurch ermöglicht es die numerische Inversionslösung, im Gegensatz zur analytischen Lösung, die Einzugsbereiche der IPV unter Berücksichtigung der Heterogenität des Untergrunds und der Einflüsse
Abb. 1 Prinzip der Immissionspumpversuche (IPV) (Ptak et al. 2004)

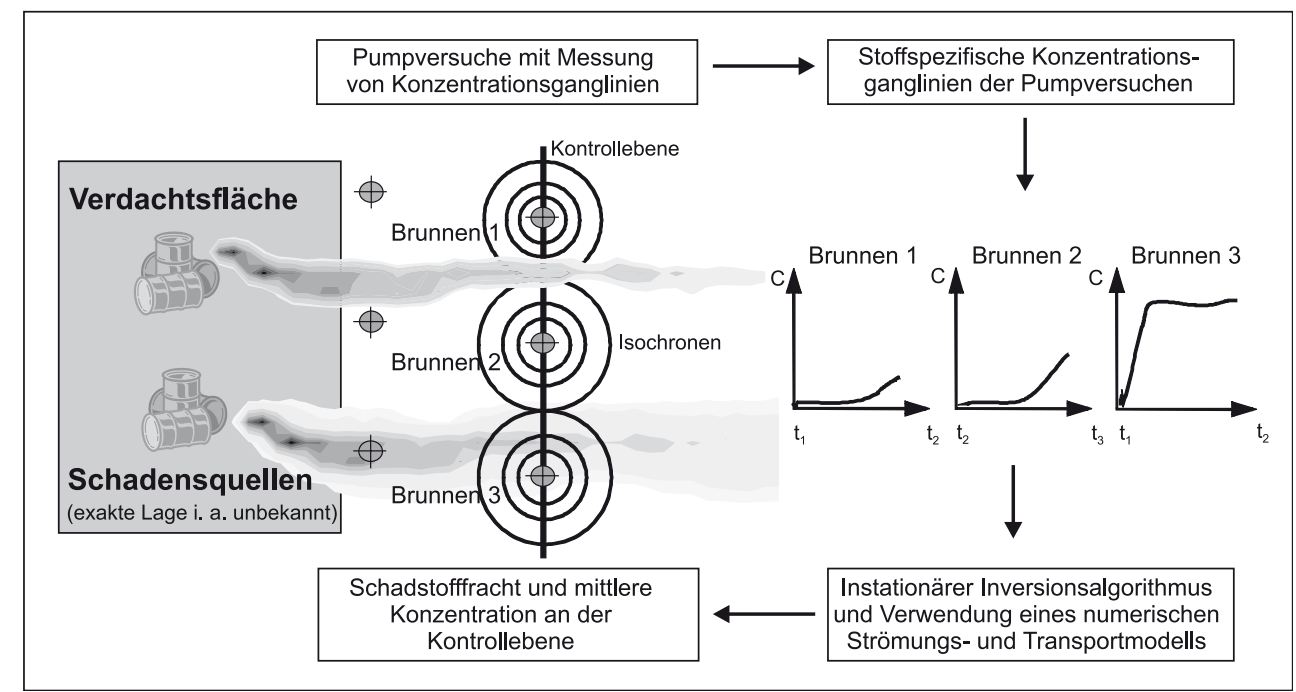


des hydraulischen Gradienten abzubilden. Die Schadstofffracht über die Kontrollebene, $M_{C P}\left[\mathrm{M} \mathrm{T}^{-1}\right]$, wird wie folgt berechnet:

$$
\text { [1] } \quad M_{C P}(t)=\int_{C P(t)} C_{0}(x, 0) q_{0 y}(x, 0) b(x, 0) d x
$$

wobei $C_{0}(x, 0)\left[\mathrm{M} \mathrm{L}^{-3}\right]$ die tiefengemittelte Konzentrationsverteilung vor der Pumpmaßnahme entlang der Kontrollebene in x-Richtung, $q_{0 y}(x, 0)\left[\mathrm{L} \mathrm{T}^{-1}\right]$ die Darcy-Geschwindigkeit vor der Pumpmaßnahme in y-Richtung senkrecht zur Kontrollebene, positioniert bei $y=0, \ell_{C P}(t)$ die Länge der durch die Isochronen der IPV erfassten Kontrollebene zum Zeitpunkt $t$ und $b(x, 0)[\mathrm{L}]$ die wassererfüllte Mächtigkeit des Grundwasserleiters darstellen. Zur Bestimmung der Konzentrationsverteilung $C_{0}(x, 0)\left[\mathrm{M} \mathrm{L}^{-3}\right]$ werden Isochronen zu Zeitpunkten $t$ der Probennahme in den Konzentrationsganglinien unter Berücksichtigung der natürlichen Grundwasserströmung im Modell berechnet. Die Abhängigkeit der Konzentrationsganglinien $C_{w}(t)\left[\mathrm{M} \mathrm{L}^{-3}\right]$ von der Konzentrationsverteilung vor Beginn der Pumpmaßnahme $C_{0}(x, y)\left[\mathrm{M} \mathrm{L}^{-3}\right]$ kann durch die folgende Massenbilanz (Bayer-Raich et al. 2004) dargestellt werden:

[2] $\quad Q_{w} C_{w}(t)=-\oint_{\ell_{l}(t)} C_{0}(x, y) \vec{q}_{w}(x, y) \vec{n} b(x, y) d \ell$

wobei $\vec{q}_{w}(x, y)\left[\mathrm{L} \mathrm{T}^{-1}\right]$ als Darcy-Geschwindigkeit während des Pumpversuchs, $\vec{n}$ als nach außen zeigender Einheitsvektor rechtwinklig zur Isochrone, $b(x, y)[\mathrm{L}]$ als wassergefüllte Aquifermächtigkeit, $Q_{w}=-\int_{\ell_{l}(t)} \vec{q}_{w}(x, y) \vec{n} b(x, y) d \ell\left[\mathrm{L}^{3} \mathrm{~T}^{-1}\right]$ als Pumprate am Brunnen und $\ell_{l}(t)$ als Isochrone zum Probennahmezeitpunkt $t$ definiert ist. Gleichung 2 wird für jeden Wert der Konzentrationsganglinie gelöst, wobei die Konzentrationsverteilung der vorausgegangenen Isochronen mit einbezogen wird. Details sind z. B. in Ptak et al. (2000), Bayer-Raich et al. (2003), Bayer-Raich (2004) und Herold et al. (2008) enthalten.

Frachtbestimmung mittels Punktkonzentrationsmessungen

Der für diese Studie verwendete Ansatz wurde von Bockelmann et al. (2003) übernommen:

$$
M_{C P}=\sum_{n=1}^{n_{\text {Brumen }}}\left(i_{n} \cdot C_{n} \cdot K_{n} \cdot A_{n}\right)
$$

wobei $M_{C P}$ die Schadstofffracht an der Kontrollebene, $i_{n}$ den Gradienten an der Messstelle $n, C_{n}$ die Konzentration an der Messstelle, $K_{n}$ den hydraulischen Durchlässigkeitsbeiwert an der Messstelle und $A_{n}$ die Fläche, die durch die Probe bzw. Messstelle $n$ repräsentiert wird, beschreiben. Bockelmann et al. (2003) wählten diesen Ansatz, da alle Messstellen voll verfiltert waren und keine Multi-Level-Daten zur Verfügung standen. In dieser Studie handelt es sich um dasselbe Testgelände, und wiederum standen nur tiefenintegrierende Proben zur Verfügung.
Bei der Auswertung wurde Wert darauf gelegt, dass die Gesamtsumme ( $\left.\sum_{n=1}^{n_{\text {Bnumen }}} A_{n}\right)$ der IPV- Kontrollebenenflächenanteile sowie die Summe der für die Punktkonzentrationsmessungen repräsentativen Teilflächen identisch sind. Die Konzentrationsganglinien wurden daher so gekürzt, dass es zu keiner Überlappung der Einzugsbereiche von nacheinander durchgeführten IPV kam.

\section{Durchführung}

Im Jahr 2006 wurden sechs IPV an den Brunnen der Kontrollebene CP2-3 (B97-B102, s. Abb. 2) innerhalb eines Zeitraums von 42 Tagen durchgeführt. Um gegenseitige Beeinflussung zu vermeiden, wurden nur maximal 2 Brunnen gleichzeitig bepumpt. Ebenso wurde eine möglichst lange Wiederanstiegsphase zwischen den Pumpkampagnen gewählt (3 Tage), um eine Wiederherstellung des natürlichen Strömungsfeldes zu ermöglichen, was durch fortlaufende Wasserstandsmessungen kontrolliert wurde.

Für die Punktkonzentrationsmessungen wurden 13 Direct-Push-Messstellen im Abstand von 3 bis 11 Meter zwischen den Brunnen B97 und B100 errichtet (Abb. 2). Der Vergleich der Schadstoffmassenfrachten aus den IPV und der Interpolation von Punktkonzentrationswerten erfolgt somit an einem Teilstück der Kontrollebene CP2-3. Die Probennahmezeitpunkte während der Pumpdauer an den IPV-Brunnen von jeweils rund 100 Stunden ergaben einen Abstand zwischen den Isochronen von $2 \mathrm{~m}$, was eine höhere räumliche Auflösung darstellt als bei den Direct-PushMessstellen.

Die Beprobung der Direct-Push-Messstellen erfolgte über Membranpumpen, wobei der Temperaturverlauf, die elektrische Leitfähigkeit, der pH-Wert, das Redoxpotenzial und die Konzentration an gelöstem Sauerstoff mithilfe einer Durchflusszelle im Förderstrom bestimmt wurden. Die Probennahme von zwei Litern Grundwasser erfolgte, nachdem das zweifache Brunnenvolumen abgepumpt worden war. Die Analyse der Grundwasserproben aus den IPV und aus den Direct-PushMessstellen wird bei Herold et al. (2008) beschrieben.

\section{Modellierung von Strömung und Transport}

Das in dieser Studie zur Inversion der IPV-Konzentrationsganglinien mittels CSTREAM (Bayer-Raich et al. 2003, Bayer-Raich 2004) verwendete numerische Strömungs- und Transportmodell basiert auf den Modellen von Bösel et al. (2000), Jarsjö et al. (2005) und Mak et al. (2006) und wurde in Richtung Norden erweitert, um die Schadstofffahne und alle Kontrollebenen abzudecken. Es handelt sich um ein mit MODFLOW (Harbaugh \& McDonald 1996) und MODPATH (Pollock 1994) erstelltes zweidimensionales 


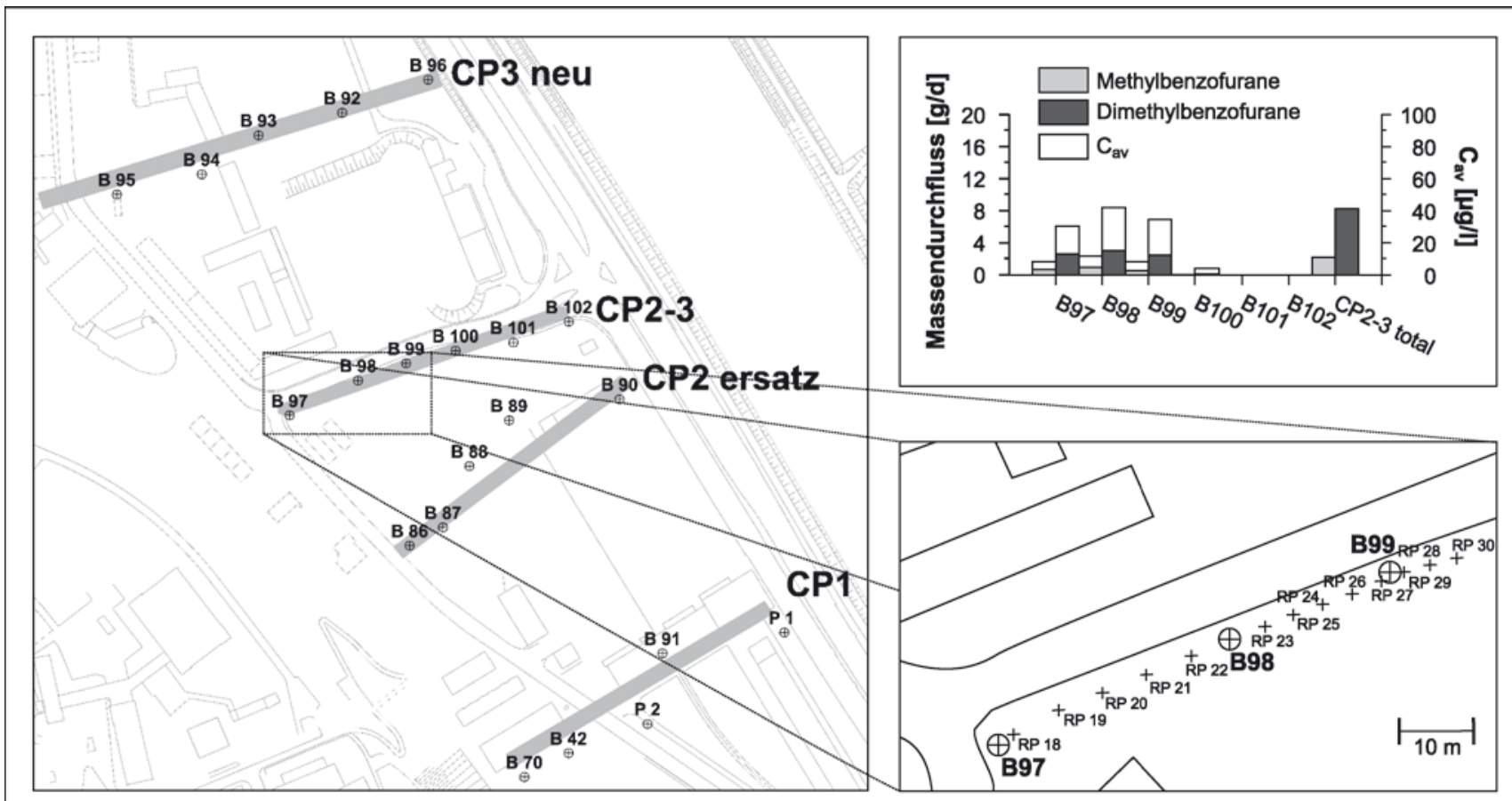

Abb. 2 Position der Kontrollebenen und Brunnen im Testfeld Süd (links), Position der Direct-Push-Messstellen zur Bestimmung von Punktkonzentrationswerten (rechts unten), Schadstoffmassenfrachten und mittlere Konzentration $\left(\mathrm{C}_{\mathrm{av}}\right)$ an Kontrollebene CP2-3 (IPV-spezifische Auftragung der Frachten) (rechts oben)

Modell. Ein 2D-Ansatz wurde gewählt, da der Grundwasserleiter geringmächtig und die Brunnen voll verfiltert sind. Die verwendeten Randbedingungen sind in Herold et al. (2008) dargestellt. Potenzialmessungen im Feld (im Rahmen von Stichtagsmessungen) ergaben nur geringe Grundwasserstands- und Fließrichtungsänderungen, was sich auf die versiegelte Oberfläche im Untersuchungsgebiet und den regulierenden Einfluss des gestauten Vorfluters zurückführen lässt. Dies führt zu der Annahme, dass es sich um ein stationäres System handelt. Weitere Details zum Modell und zur Auswertung mit CSTREAM sind in Herold et al. (2008) enthalten.

\section{Ergebnisse}

Tabelle 1 stellt die ermittelten Schadstofffrachten an dem untersuchten Teilstück der Kontrollebene (CP2-3) für die im Testfeld Süd dominierenden Schadstoffkomponenten dar, die (i) mittels Interpolation von Punktkonzentrationsmessungen entsprechend Gleichung 3 und (ii) mithilfe des Programms CSTREAM und des numerischen Standortmodells für das Gelände berechnet wurden. Die für die Interpolation von Punktkonzentrationsmessungen verwendeten Daten sind in Tabelle 2 dargestellt, wobei die hydraulischen Durchlässigkeitsbeiwerte an den Rammpegeln durch In- terpolation der pumpversuchsbasierten Werte an den IPVBrunnen bestimmt wurden.

Es ist deutlich zu erkennen, dass die Gesamtschadstofffrachten der zwei HET (Methylbenzofurane und Dimethylbenzofurane), unabhängig von der Methode, jeweils ähnliche Ergebnisse aufweisen. Die Gesamtschadstofffracht der Methylbenzofurane beträgt 1,36 bzw. 1,38 g/d und die der Dimethylbenzofurane 5,22 und 5,34 g/d, wobei die absoluten Unterschiede $(0,02$ und $0,12 \mathrm{~g} / \mathrm{d})$ und auch die relativen Abweichungen $(-1,45 \%$ und $-2,25 \%)$ minimal ausfallen.

Ganz anders stellt sich das Bild bei Acenaphthen dar. Hier beträgt der quantitative Unterschied der durch die zwei unterschiedlichen Methoden bestimmten Gesamtschadstofffrachten 1,68 g/d und auch die relative Abweichung $(-25,23 \%)$ ist erheblich größer.

Abbildung 3 stellt die Daten aus Tabelle 2 dar, wobei deutlich wird, dass beide Methoden das Fahnenzentrum hervorheben und einen erneuten Anstieg der Schadstofffrachten am westlichen Rand verzeichnen (vgl. Herold et al. 2008). Aus den Daten geht hervor, dass die Summe der aus Punktkonzentrationsmessungen berechneten Schadstofffrachten der zwei HET (Methylbenzofurane und Dimethylbenzofurane) im Erfassungsbereich des IPV am Brunnen B97 einen relativen Anstieg von $43 \%$ bzw. 50\% gegenüber dem entsprechenden IPV-Ergebnis aufweisen, wohingegen die Summe der Punktkonzentrationsmessungen im Erfassungs- 
Tab. 1 Vergleich der Schadstoffmassenflüsse entlang Kontrollebene CP2-3

\begin{tabular}{|c|c|c|c|c|c|c|}
\hline & \multicolumn{2}{|c|}{ Methylbenzofurane } & \multicolumn{2}{|c|}{ Dimethylbenzofurane } & \multicolumn{2}{|c|}{ Acenaphthen } \\
\hline & $\begin{array}{c}\mathrm{MF}[\mathrm{g} / \mathrm{d}] \\
\text { Punkt }\end{array}$ & $\begin{array}{c}\mathrm{MF}[\mathrm{g} / \mathrm{d}] \\
\mathrm{IPV}\end{array}$ & $\begin{array}{c}\mathrm{MF}[\mathrm{g} / \mathrm{d}] \\
\text { Punkt }\end{array}$ & $\begin{array}{c}\mathrm{MF}[\mathrm{g} / \mathrm{d}] \\
\mathrm{IPV}\end{array}$ & $\begin{array}{c}\mathrm{MF}[\mathrm{g} / \mathrm{d}] \\
\text { Punkt }\end{array}$ & $\begin{array}{c}\mathrm{MF}[\mathrm{g} / \mathrm{d}] \\
\mathrm{IPV}\end{array}$ \\
\hline B97 & 0,34 & 0,42 & 1,28 & 1,54 & 0,35 & 1,38 \\
\hline RP18 & 0,09 & & 0,33 & & 0,17 & \\
\hline RP19 & 0,06 & & 0,24 & & 0,05 & \\
\hline RP20 & 0,11 & & 0,46 & & 0,70 & \\
\hline RP21 & 0,14 & & 0,51 & & 0,88 & \\
\hline RP22 & 0,14 & & 0,46 & & 0,38 & \\
\hline B98 & 0,08 & 0,67 & 0,31 & 2,43 & 0,91 & 4,34 \\
\hline $\mathrm{RP} 23$ & 0,06 & & 0,20 & & 0,35 & \\
\hline RP24 & 0,07 & & 0,30 & & 0,43 & \\
\hline $\mathrm{RP} 25$ & 0,08 & & 0,32 & & 0,41 & \\
\hline RP26 & 0,07 & & 0,28 & & 0,12 & \\
\hline $\mathrm{RP} 27$ & 0,06 & & 0,21 & & 0,16 & \\
\hline B99 & 0,03 & 0,28 & 0,11 & 1,44 & 0,08 & 0,94 \\
\hline RP28 & 0,02 & & 0,09 & & 0,00 & \\
\hline RP29 & 0,01 & & 0,07 & & 0,00 & \\
\hline RP30 & 0,00 & & 0,01 & & 0,00 & \\
\hline $\mathrm{B} 100$ & 0,01 & 0,00 & 0,05 & 0,02 & 0,00 & 0,00 \\
\hline Summe & 1,36 & 1,38 & 5,22 & 5,34 & 4,98 & 6,66 \\
\hline
\end{tabular}

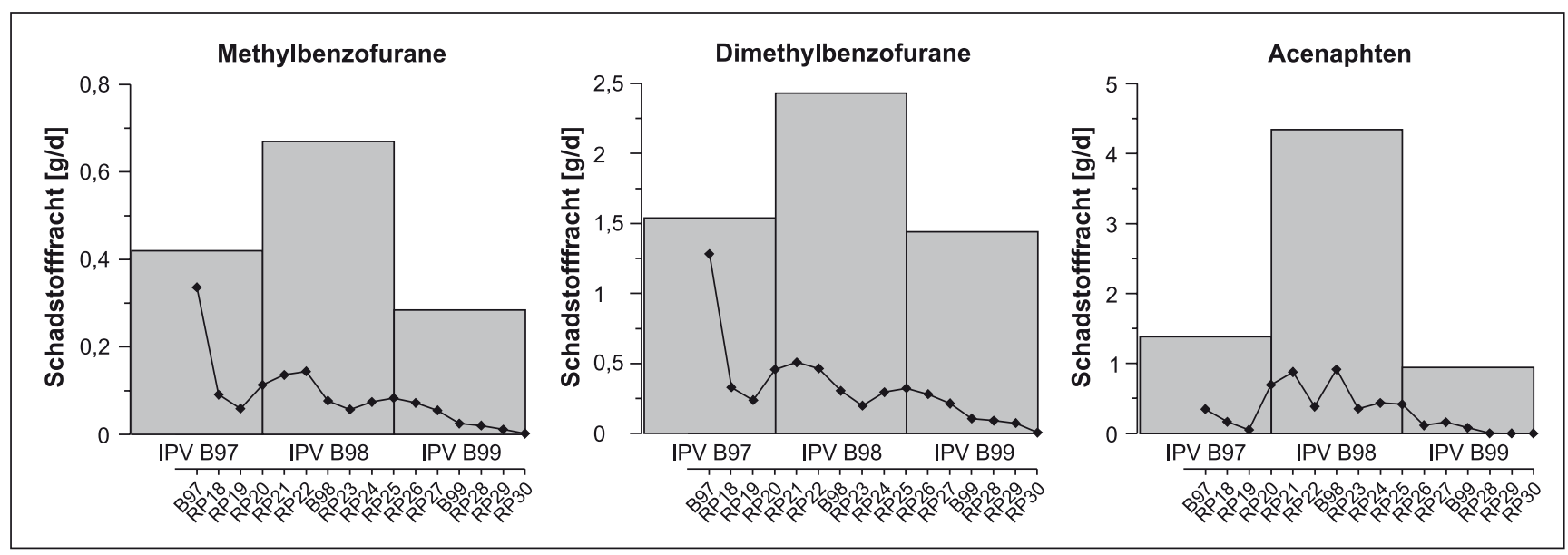

Abb. 3 Schadstoffmassenfrachten für Methylbenzofurane, Dimethylbenzofurane und Acenaphthen: Bestimmung mit IPV (graue Balken, Breite entspricht Einzugsbereich) und über Punktkonzentrationsmessungen (schwarze Linie)

bereich der anderen zwei IPV (B98 und B99) eine relative Abnahme verzeichnen. Das legt die Vermutung nahe, dass die für Brunnen B97 aus der Interpolation berechnete außergewöhnlich hohe Schadstofffracht ein von einer einzelnen Schadstoffkonzentration überprägtes Bild darstellt. Die Variabilität der Schadstoffmassenfrachten an den Punktmessstellen nimmt in Richtung Osten ab, wobei die im Einzugsbereich des IPV an Brunnen B97 summierten ungewöhnlich hohen Schadstofffrachten durch eine relative Abnahme im Einzugsbereich der anderen beiden IPV, über die Kontrollebene betrachtet, ausgeglichen werden.
Die Ursache für die Unterschiede der Gesamtschadstofffracht bei Acenaphthen wird deutlich, wenn die während der IPV gemessenen Konzentrationsganglinien betrachtet werden (Abb. 4). Am Beispiel der Abbildung 4 ist zu erkennen, dass die HET-Konzentrationsganglinien einen nahezu konstanten Verlauf aufweisen, wobei Methylbenzofuran im Vergleich zu Dimethylbenzofuran eine geringere Schwankungsbreite zeigt. Im Gegensatz dazu variiert die Konzentrationsganglinie von Acenaphthen über den betrachteten Zeitraum von 99 Stunden zwischen 110 und $70 \mu \mathrm{g} / \mathrm{l}$, d.h. mit einer deutlich größeren Schwankungsbreite. Entspre- 
Tab. 2 Daten für die Interpolation der Punktkonzentrationsmessungen $\left(\mathrm{C}_{\mathrm{ACE}}=\right.$ Konzentration Acenaphthen, $\mathrm{C}_{\mathrm{MBF}}=$ Konzentration Methylbenzofurane, $\mathrm{C}_{\mathrm{DMBF}}=$ Konzentration Dimethylbenzofurane, $\mathrm{K}_{\mathrm{f}}=$ hydraulischer Durchlässigkeitsbeiwert, $\mathrm{A}=$ Fläche und $\mathrm{i}=\mathrm{Gradient}$ ).

\begin{tabular}{|c|c|c|c|c|c|c|}
\hline & $\mathrm{C}_{\mathrm{ACE}}[\mu \mathrm{g} / \mathrm{l}]$ & $\mathrm{C}_{\mathrm{MBF}}[\mu \mathrm{g} / \mathrm{I}]$ & $\mathrm{C}_{\mathrm{DMBF}}[\mu \mathrm{g} / \mathrm{l}]$ & $K_{f}[m / s]$ & $\mathbf{A}\left[\mathbf{m}^{2}\right]$ & i $[-]$ \\
\hline B97 & 12 & 11,7 & 44,5 & $2,84 \mathrm{E}-03$ & 48,96 & 0,0024 \\
\hline RP18 & 23 & 12,6 & 45,9 & $3,01 \mathrm{E}-03$ & 11,53 & 0,0024 \\
\hline RP19 & 4,9 & 5,7 & 22,7 & $3,18 \mathrm{E}-03$ & 15,87 & 0,0024 \\
\hline RP20 & 65 & 10,5 & 42,6 & $3,34 \mathrm{E}-03$ & 15,44 & 0,0024 \\
\hline RP21 & 80 & 12,4 & 46,4 & $3,51 \mathrm{E}-03$ & 15,04 & 0,0024 \\
\hline RP22 & 35 & 13,1 & 42,4 & $3,68 \mathrm{E}-03$ & 14,36 & 0,0024 \\
\hline B98 & 98,1 & 8,3 & 32,8 & $3,85 \mathrm{E}-03$ & 11,65 & 0,0024 \\
\hline RP23 & 45 & 7,3 & 25,5 & $3,60 \mathrm{E}-03$ & 10,43 & 0,0024 \\
\hline RP24 & 55 & 9,4 & 37,6 & $3,35 \mathrm{E}-03$ & 11,32 & 0,0024 \\
\hline RP25 & 52 & 10,4 & 40,7 & $3,11 \mathrm{E}-03$ & 12,32 & 0,0024 \\
\hline RP26 & 14 & 9,0 & 35,3 & $2,86 \mathrm{E}-03$ & 13,46 & 0,0024 \\
\hline RP27 & 30 & 10,4 & 40,5 & $2,61 \mathrm{E}-03$ & 9,77 & 0,0024 \\
\hline B99 & 26,7 & 8,5 & 35,8 & $2,36 \mathrm{E}-03$ & 6,08 & 0,0024 \\
\hline RP28 & 0,19 & 4,2 & 19,3 & $2,40 \mathrm{E}-03$ & 9,59 & 0,0024 \\
\hline RP29 & 0,01 & 1,9 & 12,2 & $2,43 \mathrm{E}-03$ & 11,91 & 0,0024 \\
\hline RP30 & 0,01 & 0,1 & 0,5 & $2,47 \mathrm{E}-03$ & 26,12 & 0,0024 \\
\hline B100 & 0,03 & 0,4 & 3,1 & $2,50 \mathrm{E}-03$ & 28,21 & 0,0024 \\
\hline
\end{tabular}

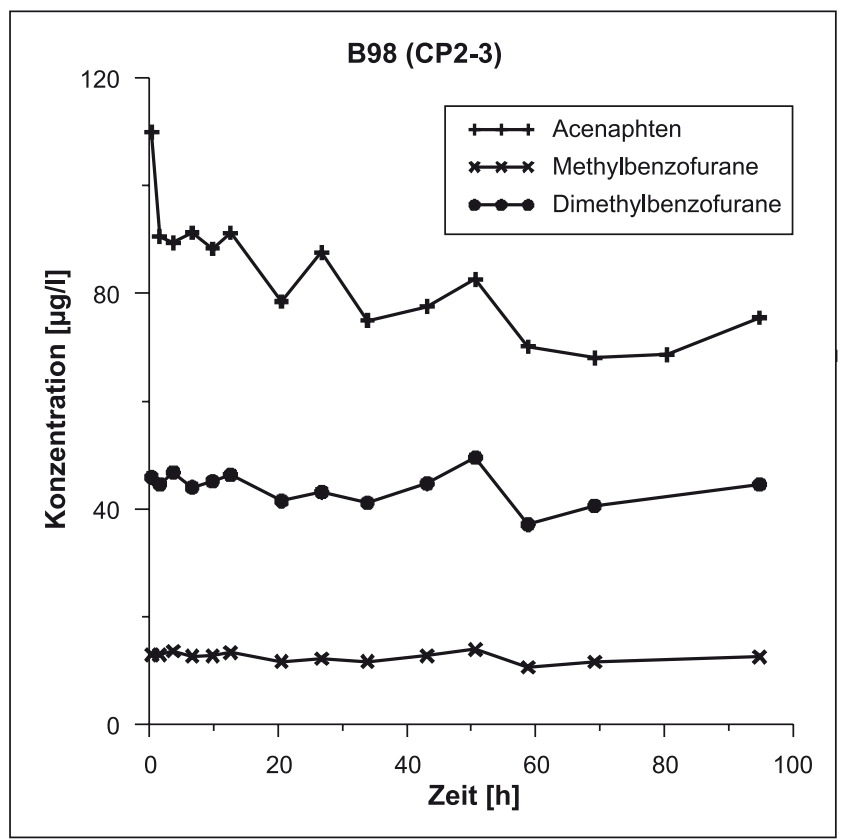

Abb. 4 Konzentrationsganglinien von Methylbenzofuranen, Dimethylbenzofuranen und Acenaphthen am Beispiel des IPV-Brunnens B98

chend den theoretischen Überlegungen zur Auswertung von Konzentrationsganglinien aus Immissionspumpversuchen (z. B. Ptak et al. 2000, Bockelmann et al. 2001, Bayer-Raich et al. 2004) indiziert eine relativ große Schwankungsbreite in der Konzentrationsganglinie eine relativ heterogene Verteilung der Schadstoffmasse innerhalb der Schadstofffahne. In diesem Fall werden für eine zuverlässige Quantifizierung der Schadstofffracht, im Vergleich zu einer Konzentrations- ganglinie mit konstantem Konzentrationsverlauf, relativ mehr Punktkonzentrationsmessungen benötigt.

Die relative Größe der Unterschiede der Schadstofffrachten aus der Interpolation von Punktkonzentrationsmessungen und aus den Immissionspumpversuchen bestätigt dieses Postulat. Für Methylbenzofuran beträgt der Unterschied der Frachten nur 0,02 g/d, hier ist die Konzentrationsganglinie nahezu konstant (s. Abb. 4). Für Dimethylbenzofuran ist im Vergleich zu Methylbenzofuran der Unterschied mit 0,12 g/d etwas größer, ebenso wie die Schwankungsbreite der Dimethylbenzofuran-Konzentrationsganglinie. Dagegen führt die große Schwankungsbreite der Acenaphthen-Konzentrationsganglinie und entsprechend die große räumliche Variabilität der Acenaphthen-Masse innerhalb der Schadstofffahne dazu, dass mit der Interpolation von Punktkonzentrationsmessungen die Gesamtfracht im vorliegenden Fall deutlich unterschätzt wird (4,98 g/d für die Frachtbestimmung mittels Punktkonzentrationsmessungen im Gegensatz zu 6,66 g/d für die IPV). Die Ergebnisse des Vergleichs legen nahe, dass das Direct-Push-Messstellennetz trotz des relativ geringen Messstellenabstandes von bis zu $3 \mathrm{~m}$ noch nicht dicht genug war, um die Verteilung der Acenaphthen-Masse im Aquifer mittels Interpolation von Punktkonzentrationswerten hinreichend genau quantifizieren zu können. Offensichtlich konnten mit den Punktkonzentrationsmessungen einzelne Bereiche relativ hoher Konzentrationen nicht erfasst werden. Die Bestimmung der Anzahl der erforderlichen Punktkonzentrationsmesswerte als Funktion einer zulässigen Abweichung der Ergebnisse aus den beiden Frachtbestimmungsmethoden war nicht Inhalt der durchgeführten Untersuchungen. Sie ließe sich z. B. über eine Szenariomodellierung zur Verdichtung des Messstellennetzes unter Verwendung der aus 
den IPVs ermittelten räumlichen Konzentrationsverteilung ableiten, oder aber durch eine systematische Verdichtung des Messstellennetzes im Testfeld bestimmen. Es sei hier angemerkt, dass bereits die im Testfeld jetzt implementierte Messstellendichte unter Praxisbedingungen in der Regel wohl nicht realisierbar wäre.

Eine Multilevelbeprobung in den Messstellen zur Gewinnung von tiefenorientierten Punktkonzentrationsmesswerten und deren anschließenden Interpolation (3D-Ansatz) würde die Situation nicht zwingend verbessern, da auch in diesem Fall der Aquiferbereich zwischen den Messstellen, im Gegensatz zum IPV, nicht erfasst werden würde. Auch würde der 3D-Ansatz einen unter Praxisbedingungen in der Regel nicht vertretbaren Erkundungsaufwand (tiefenorientierte Bestimmung von $\mathrm{K}_{\mathrm{f}}$-Werten, hydraulischen Gradienten, Analytik etc.) erfordern.

Aus den beiden Methoden ergeben sich Unsicherheiten, welche ebenfalls die Ergebnisse beeinflussen können. Die Ergebnisunsicherheit der IPV sowie der Frachtbestimmung über Punktkonzentrationsmessungen ergibt sich aus der Analytik und aus der Anwendung physikalischer Messverfahren. Für die Analytik von Acanaphthen, Methylbenzofuranen und Dimethylbenzofuranen ergibt sich jeweils eine Unsicherheit von 5\%. Die Unsicherheit aufgrund von physikalischen Messungen beträgt in beiden Fällen etwa 10\%. Die den Punktkonzentrationsmessungen inhärente Unsicherheit infolge der Interpolation wird bei der IPV-Methode durch die integrale Natur der Methode aufgehoben. Retardation kann bei den IPV berücksichtigt werden (Bayer-Raich et al. 2006, Herold et al. 2008). Es stellt sich auch die Frage inwieweit Konzentrationsgradienten, z.B. hervorgerufen durch NA, das Ergebnis der IPV beeinflussen können. Jarsjö \& Bayer-Raich (2008) haben nachgewiesen, dass konstante Konzentrationsgradienten in Richtung der Grundwasserströmung keinen nennenswerten Effekt auf das Ergebnis haben. Somit kann gefolgert werden, dass die Unterschiede bei der Frachtbestimmung mittels der beiden Methoden nicht durch Messfehler bzw. Fehler aufgrund vereinfachender Annahmen bei der IPV-Auswertung dominiert werden.

\section{Schlussfolgerungen}

Der hier vorgestellte Vergleich von Schadstofffrachten aus Immissionspumpversuchen und aus der Interpolation von Punktkonzentrationsmessungen hat gezeigt, dass auch hochaufgelöste Punktkonzentrationsmessungen je nach Standortsituation und Heterogenität der Schadstoffverteilung im Untergrund zu Unsicherheiten bei der Frachtbestimmung führen können. Die Erkundungsunsicherheit nimmt dabei mit dem Grad der Heterogenität der Schadstoffverteilung zu, da die Wahrscheinlichkeit, Bereiche mit relativ hoher Schadstoffkonzentration zu verfehlen oder aber hohe Konzentra- tionswerte durch die Interpolation über in Wirklichkeit gar nicht oder nur wenig kontaminierte Bereiche zu verteilen, zunimmt. Ein dichteres Direct-Push-Messstellennetz, bei dem Schadstoffkonzentrationen und hydraulische Durchlässigkeitsbeiwerte ggf. auch vertikal aufgelöst gemessen werden, könnte die Verlässlichkeit der Frachtbestimmung basierend auf Punktkonzentrationsmessungen erhöhen, wobei allerdings die Limitierungen bei der Verfügbarkeit von Bohransatzpunkten und die zu erwartenden Installationsund Beprobungskosten berücksichtigt werden müssten. Hier können Immissionspumpversuche aufgrund des mit der Zeit zunehmenden Probennahmevolumens und ihrer integrierenden Wirkung einen wichtigen Beitrag zur sicheren Bestimmung von Schadstofffrachten und Konzentrationsverteilungen entlang von Kontrollebenen leisten, auch an Standorten mit eingeschränkter Zugänglichkeit, z.B. infolge von Bebauung und Nutzung. Dabei hilft die Anwendung eines numerischen Strömungs- und Transportmodells, durch das es möglich wird, auch hydraulisch und hydrogeochemisch heterogene Standortbedingungen und reaktiven Transport zu simulieren. Die Erhöhung der Erkundungsgenauigkeit ist insbesondere bei der Standortbeurteilung sowie bei der Planung und Durchführung von Sanierungsmaßnahmen von Bedeutung. Des Weiteren kann durch die gesamte Erfassung des mobilen Anteils an Schadstoffen im Einzugsgebiet eines IPV-Brunnens bestimmt werden, welche Schadstoffe aufgrund ihrer Mobilität priorisiert werden müssen. Letztendlich kann die erhöhte Erkundungssicherheit auch eine genauere Beurteilung der Effizienz von bereits implementierten Sanierungsmaßnahmen ermöglichen.

Danksagung Das diesem Bericht zugrundeliegende Vorhaben wurde mit Mitteln des Bundesministeriums für Bildung und Forschung (BMBF) unter dem Förderkennzeichen 02WN0361 im Rahmen des Projektverbundes KORA gefördert. Die Autoren bedanken sich für die Finanzierung durch das BMBF sowie für die freundliche Unterstützung durch das AfU Stuttgart, die EnBW, die Helmholtz-Zentrum für Umweltforschung GmbH - UFZ (Martí Bayer-Raich) und die Firma Büro Holder sowie bei Herrn Matthias Piepenbrink für seine Mitarbeit in diesem Projekt.

Open Access Dieser Artikel wird zu den Bedingungen der „Creative Commons Attribution Noncommercial License“ zur Verfügung gestellt. Damit ist eine nichtkommerzielle Nutzung, Verbreitung und Vervielfältigung erlaubt, sofern die Autoren des Artikels und die genaue Quelle angegeben sind.

\section{Literatur}

Ballard, S.: The in situ permeable flow sensor: a ground-water flow velocity meter.- Ground Water 34(2), 231-240 (1996)

Bauer, S., Bayer-Raich, M., Holder, T., Kolesar, C., Müller, D., Ptak, T.: Quantification of groundwater contamination in an urban area using integral pumping tests.- J. Contam. Hydrol. 75, 183-213 (2004)

Bayer-Raich, M.: Integral pumping tests for the characterization of groundwater contamination.- $\mathrm{PhD}$ Thesis, Center for Applied Geoscience, Universität Tübingen, Tübingen, 112 S. (2004) 
Bayer-Raich, M., Jarsjö, J., Holder, T., Ptak, T.: Numerical estimations of contaminant mass flow rate based on concentration measurements in pumping wells. Calibration and reliability in groundwater modelling: A few steps closer to reality (Proceedings of ModelCARE'2002, Prague, Czech Republic, June 2002) IAHS Publ. 277, 10-16 (2003)

Bayer-Raich, M., Jarsjö, J., Liedl, R., Ptak, T., Teutsch, G.: Average contaminant concentration and mass flow in aquifers from time dependent pumping well data: analytical framework.- Water Resour. Res. 40, W08303. doi:10.1029/2004/WR003095 (2004)

Bayer-Raich, M., Jarsjö, J., Liedl, R., Ptak, T., Teutsch, G.: Integral pumping test analyses of linearly sorbed groundwater contaminants using multible wells: Inferring mass flows and Natural Attenuation rates.- Water Resour. Res. 42, W08411. doi:10.1029/2005WR004244 (2006)

Bayer-Raich, M., Jarsjö, J., Teutsch, G.: Comment on "Analysis of groundwater contamination using concentration-time series recorded during an integral pumping test: Bias introduced by strong concentration gradients within the plume" by Allelign Zeru and Gerhard Schäfer.- J. Contam. Hydrol. 90, 240-251 (2007)

Bockelmann, A., Ptak, T., Teutsch, G.: An analytical quantification of mass fluxes and natural attenuation rate constants at a former gasworks site.- J. Contam. Hydrol. 53(3-4), 429-453 (2001)

Bockelmann, A., Zamfirescu, D., Ptak, T., Grathwohl, P., Teutsch, G.: Quantification of mass fluxes and natural attenuation rates at an industrial site with a limited monitoring network: a case study.J. Contam. Hydrol. 60, 97-121 (2003)

Bösel, D., Herfort, M., Ptak, T., Teutsch, G.: Design, performance, evaluation and modelling of a natural gradient multitracer transport experiment in a contaminated heterogeneous porous aquifer.- In: Dassargues, A. (Hrsg.) Tracers and Modelling in Hydrogeology.IAHS Publication 262, 45-51, Liege (2000)

Borden, R.C., Daniel, R.A., Lebrun IV, L.E., Davis, C. W.: Intrinsic biodegradation of MTBE and BTEX in a gasoline-contaminated aquifer.- Water Resour. Res. 33(5), 1105-1115 (1997)

Einarson, M.D., Mackay, D. M.: Predicting impacts of groundwater contamination. Environ.- Sci. Technol. 35(3), 66A-73A (2001)

Harbaugh, A.W., McDonald, M.G.: User's documentation for MODFLOW-96, an update to the U.S. Geological Survey modular finite-difference ground-water flow model.- U.S. Geological Survey Open-File Report 96-485 (1996)

Hatfield, K., Annable, M.D., Kuhn, S., Rao, P. S.C., Cambell, T.: In: Thornton, S.F., Oswald, S.E. (Hrsg.) A new method for quantifying contaminant flux at hazardous waste sites, in groundwater quality: natural and enhanced restoration of groundwater pollution.- IAHS Publication 275, 25-31 (2002)

Hatfield, K., Annable, M., Cho, J., Rao, P. S. C., Klammler, H.: A direct passive method for measuring water and contaminant fluxes in porous media.- J. Contam. Hydrol. 75, 155-181 (2004)

Herfort, M., Ptak, T., Hümmer, O., Teutsch, G., Dahmke A.: Testfeld Süd: Einrichtung der Testfeldinfrastruktur und Erkundung hydraulisch-hydrogeochemischer Parameter des Grundwasserleiters.- Grundwasser 3(4), 159-166 (1998)

Herfort, M.: Reactive transport of organic compounds within a heterogeneous porous aquifer.- $\mathrm{PhD}$ Thesis, Universität Tübingen, Tübingen, 59 S. (2000)

Herold, M., Ptak, T., Wendel, T., Grathwohl, P.: Integrale Quantifizierung von Schadstoffmassenflüssen im Grundwasser - Konditionierung der numerischen Inversion der Konzentrationsganglinien von Immissionspumpversuchen.- Grundwasser 13(1), 4-14 (2008). doi: 10.1007/s00767-007-0057-2

Jarsjö, J., Bayer-Raich, M., Ptak, T.: Monitoring groundwater contamination and delineating source zones at industrial sites: Uncertainty analyses using integral pumping tests.- J. Contam. Hydrol. 79, 107-134 (2005)
Jarsjö, J., Bayer-Raich, M.: Estimating plume degradation rates in aquifers: Effect of propagating measurement and methodological errors.- Water Resour. Res. 44, W02501 (2008). doi:10.1029/ 2006WR005568

Kao, C.M., Wang, Y.S.: Field investigation of the natural attenuation and intrinsic biodegradation rates at an underground storage tank site.- Environ. Geol. 40(4-5), 622-631 (2001)

King, M.W. G., Barker, J.F., Devlin, J.T., Butler, B. J.: Migration and natural fate of a coal tar creosote plume: 2 . Mass balance and biodegradation indicators.- J. Contam. Hydrol. 39, 281-307 (1999)

Kübert, M., Finkel, M.: Contaminant mass discharge estimation in groundwater based on multi-level point measurements: A numerical evaluation of expected errors.- J. Contam. Hydrol. 84, 55-80 (2006)

LABO-Positionspapier: Berücksichtigung natürlicher Schadstoffminderungsprozesse bei der Altlastenbearbeitung.- Ad-hoc Unterausschuss „Natürliche Schadstoffminderung“ des Altlastenausschuss (ALA) des Bund/Länder-Arbeitsgemeinschaft Bodenschutz (LABO) (2005)

Mak, K.S., Griebler, C., Meckenstock, R.U., Liedl, R., Peter, A.: Combined application of conservative transport modelling and compound-specific carbon isotope analyses to assess in situ attenuation of benzene, toluene, and o-xylene.- J. Contam. Hydrol. 88, 306-320 (2006)

Newell, C. J., Conner, J.A., Rowen, D.A.: Groundwater Remediation Strategies Tool.- Publication Number 4730. API American Petroleum Institute, Houston, TX, 80 S. (2003)

Pollock, D.: User's guide for MODPATH/MODPATH-PLOT, Version 3: A particle tracking post-processing package for MODFLOW, the U.S. Geological Survey finite-difference ground-water flow model.- U. S. Geological Survey (1994)

Ptak, T., Schwarz, R., Holder, T., Teutsch, G.: Ein neues integrales Verfahren zur Quantifizierung der Grundwasserimmission: II. Numerische Lösung und Anwendung in Eppelheim.- Grundwasser 5(4), 176-183 (2000)

Ptak, T., Bayer-Raich, M., Bauer, S.: Tiefenorientierte integrale Erkundung der Schadstoffbelastung in großräumig kontaminierten Aquiferen.- Grundwasser 9(4), 235-247 (2004)

Rügner, H., Holder, T., Maier, U., Bayer-Raich, M., Grathwohl, P., Teutsch, G.: Natural Attenuation-Untersuchungen „ehemalige Abfalldeponie Osterhofen“.- Grundwasser 9(2), 98-108 (2004)

Schwarz, R.: Grundwasser-Gefährdungsabschätzung durch Emissions- und Immissionsmessungen an Deponien und Altlasten.- $\mathrm{PhD}$ thesis, Center of Applied Geosciences, Universität Tübingen, Tübingen, 125 S. (2002)

Soga, K., Page, J. W.E., Illangasekare, T. H.: A review of NAPL source zone remediation efficiency and the mass flux approach.- J. Hazard. Mater. 110, 13-27 (2004)

Teutsch, G., Ptak, T., Schwarz, R., Holder, T.: Ein neues integrales Verfahren zur Quantifizierung der Grundwasserimmission: I. Theoretische Grundlagen.- Grundwasser 5(4), 170-175 (2000)

Trötschler, O., Limburg, B., Koschitzky H.-P.: Bestimmung der hydraulischen Wirksamkeit eines Grundwasserzirkulationsbrunnens „Testfeld Süd“ mittels Tracerversuchen.- Technischer Bericht Nr. 2007/017 (VEG 28), Stuttgart, Institut für Wasserbau (2007)

US-EPA: How to evaluate alternative cleanup technologies for underground storage tank sites - a guide for corrective action plan reviewers.- Solid waste and emergency response $5401 \mathrm{G}$, EPA 510-R-04-002 (2004)

Zamfirescu, D.: Release and fate of specific organic contaminants at a former gasworks site.- PhD Thesis, Universität Tübingen, Tübingen, 96 S. (2000)

Zamfirescu, D., Grathwohl, P.: Occurrence and attenuation of specific organic compounds in the groundwater plume at a former gas 